\title{
A Biased Approach to Nonlinear Robust Stability with Applications in Adaptive Control
}

\author{
M. French and W. Bian
}

\begin{abstract}
The nonlinear robust stability theory of Georgiou and Smith (IEEE Trans. Auto. Control, 42(9):1200-1229, 1997) is generalized to the case of notions of stability with bias terms. An example from adaptive control illustrates non trivial robust stability certificates for systems which the previous unbiased theory could not establish a non-zero robust stability margin. This treatment also shows that the BIBO robust stability results for adaptive controllers in French (IEEE Trans. Auto. Control, 53(2):461-478, 2008) can be refined to show preservation of biased forms of stability under gap perturbations. In the nonlinear setting, it also is shown that, in contrast to LTI systems, the problem of minimizing nominal performance is not equivalent to maximizing the robust stability margin.
\end{abstract}

\section{INTRODUCTION}

The fundamental nonlinear robust stability framework developed by Georgiou and Smith [8] considers the classical closed loop system:

$$
[P, C]: \begin{aligned}
& y_{1}=P u_{1}, \quad u_{2}=C y_{2}, \\
& u_{0}=u_{1}+u_{2}, \quad y_{0}=y_{1}+y_{2},
\end{aligned}
$$

as depicted in Figure 1, and develops a generalisation of the

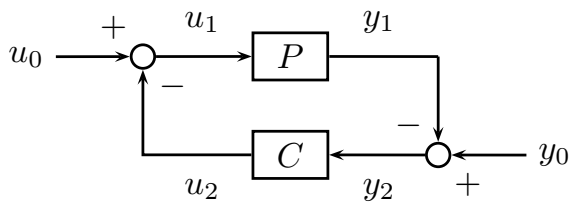

Fig. 1. The closed-loop $[P, C]$.

linear gap metric and associated robust stability results on the basis of a robust stability margin, $b_{P, C}$, which is taken to be the inverse of the induced norm of the closed loop operator $\Pi_{P / / C}$ :

$$
\Pi_{P / / C}:\left(\begin{array}{c}
u_{0} \\
y_{0}
\end{array}\right) \mapsto\left(\begin{array}{l}
u_{1} \\
y_{1}
\end{array}\right) .
$$

Under appropriate well posedness assumptions, the main robust stability theorem states that if $[P, C]$ is gain stable (that is $\left\|\Pi_{P / / C}\right\|<\infty$ ), and if

$$
\delta\left(P, P_{1}\right)<b_{P, C}:=\left\|\Pi_{P / / C}\right\|^{-1}
$$

then $\left[P_{1}, C\right]$ is gain stable $\left(\left\|\Pi_{P_{1} / / C}\right\|<\infty\right)$. Here $\delta$ denotes the nonlinear gap metric, as described later in Section 3, and is a notion of distance between plants which renders typical unmodelled dynamics small: e.g. for linear plants,

School of Electronics and Computer Science University of Southampton, Southampton SO17 1BJ, United Kingdom wb@ecs.soton.ac.uk, mcf@ecs.soton.ac.uk small multiplicative, inverse multiplicative, and co-prime factor perturbations are small in this sense, as are small time delays to proper continuous time plants (here we think of $P$ as the model, and $P_{1}$ as the 'real' system).

To account for nonlinear gains, a regional version and a gain function version of the robust stability theorem were also given. All versions of the robust stability theorem assume that the plant and the controller map zero inputs to zero outputs $(P(0)=0, C(0)=0)$ and that the closed loop operator $\Pi_{P / / C}$ has an induced norm or a gain function.

However, there are important instances in which these sufficient conditions for robust stability generically fail; and yet for which robustness results should apply and for which, to date, either relatively ad-hoc methods have been utilized to establish robust stability, or no such robust stability certificates have been established. Many such systems can be handled by developing a robust stability theory based on an underlying notion of stability which includes bias terms; for such notions of stability see [2], [10]. The first important class of examples are systems whose response depends on a non-zero initial condition, and which do not start at an equilibrium, see [7] for an alternative biased approach to such examples. The second class of systems are those for which $P(0)=0, C(0)=0$ but whose closed loop operator $\Pi_{P, C}$ is discontinuous at 0 , thus precluding the existence of a (local) finite gain. Most adaptive controllers fall within this category [3]. A third class of examples includes systems which include inherent offsets, arising e.g. from quantization errors, sensors biases etc. Another such class of feedback systems include high gain controller designs which attenuate the effects of unknown nonlinearities by nonlinear high gain feedback, and which do not cancel the effect of the nonlinearities.

In this paper, we take an important class of examples from adaptive control to motivate the approach. In this setting, the need for a bias does not arise from an offset from a single specified trajectory, so the approach of [7] is not applicable. For this class of adaptive controllers we interpret the known BIBO robust stability results of [4] to provide an interpretation based on biased stability. This approach provides a much more satisfactory approach to the underlying theory than the relatively ad-hoc techniques developed in [4], and shows the stronger result that stability with bias is preserved under sufficiently small gap perturbations.

The remainder of the paper is structured as follows. In Section 2 we introduce the system theoretic setting and notation. In Section 3 we introduce the gap metric and the notions of the robust stability margin and nominal and robust performance. Section 4 considers the motivating example from adaptive control whereby it is shown that biases are present. Section 5 establishes the main regional robust stabil- 
ity result. In Section 6 we consider the result specialised to the case of linear plants, before revisiting the adaptive control theory in Section 7. In Section 8 we consider the special case of global robust stability and the implications of the robust stability result for the formulation of appropriate optimization problems in nonlinear control design. We draw conclusions in Section 9. Due to space consideration, some proofs are omitted.

\section{SySTEMS}

The material in this section is based on [4], [5], [6], [8].

Let $\mathcal{T}$ denote either the discrete half-axis time set $\mathbb{N}$ or the continuous time counterpart, $\mathbb{R}_{+}$. For $\omega \in \mathcal{T} \cup\{\infty\}$, let $\mathcal{S}_{\omega}$ denote the set of all locally integrable maps $[0, \omega) \rightarrow \mathcal{X}$ where $\mathcal{X}$ is a nonempty set. For ease of notation define $\mathcal{S}:=\mathcal{S}_{\infty}$. For $\tau \in \mathcal{T}, \omega \in \mathcal{T} \cup\{\infty\}, 0<\tau<\omega$ define a truncation operator $T_{\tau}: \mathcal{S}_{\tau} \rightarrow \mathcal{S}$ and a restriction operator $R_{\tau}: \mathcal{S}_{\omega} \rightarrow \mathcal{S}_{\tau}$ as follows:

$$
\begin{aligned}
& T_{\tau} v(t)=\left\{\begin{array}{cc}
v(t), & t \in[0, \tau) \\
0, & \text { otherwise }
\end{array},\right. \\
& R_{\tau} v(t)=v(t), \quad t \in[0, \tau) .
\end{aligned}
$$

We define $\mathcal{V} \subset \mathcal{S}$ to be a signal space if, and only if, it is a vector space. Suppose additionally that $\mathcal{V}$ is a normed vector space and that the norm $\|\cdot\|=\|\cdot\|_{\mathcal{V}}$ is (also) defined for signals of the form $T_{\tau} v, v \in \mathcal{V}_{\tau}, \tau>0$. We can define a norm $\|\cdot\|_{\tau}$ on $\mathcal{S}_{\tau}$ by $\|v\|_{\tau}=\left\|T_{\tau} v\right\|$, for $v \in \mathcal{S}_{\tau}$. We associate spaces as follows:

- $\mathcal{V}[0, \tau)=\left\{v \in \mathcal{S}_{\tau} \mid v=R_{\tau} w, w \in \mathcal{V},\|v\|_{\tau}<\infty\right\}$, the interval space;

- $\mathcal{V}_{e}=\left\{v \in \mathcal{S} \mid \forall \tau>0: R_{\tau} v \in \mathcal{V}[0, \tau]\right\}$, the extended space;

- $\mathcal{V}_{\omega}=\left\{v \in \mathcal{S}_{\omega} \mid \forall \tau \in(0, \omega): R_{\tau} v \in \mathcal{V}[0, \tau)\right\}$, for $0<$ $\omega \leq \infty$; and

- $\mathcal{V}_{a}=\bigcup_{\omega \in(0, \infty]} \mathcal{V}_{\omega}$, the ambient space.

A signal space $\mathcal{V}$ is said to be truncation complete if $V[0, \tau)$ is complete for all $0<\tau<\infty$.

A mapping $Q: \mathcal{U}_{a} \rightarrow \mathcal{Y}_{a}$ is said to be causal if for all $x, y \in \mathcal{U}_{a}$ and $\tau \in \operatorname{dom}(x) \cap \operatorname{dom}(y)$

$$
R_{\tau} x=R_{\tau} y \Rightarrow R_{\tau}(Q x)=R_{\tau}(Q y) .
$$

Let $P: \mathcal{U}_{a} \rightarrow \mathcal{Y}_{a}$ and $C: \mathcal{Y}_{a} \rightarrow \mathcal{U}_{a}$ be causal mappings representing the plant and the controller, respectively and consider the system of equations (1.1) corresponding to Figure 1. For $w_{0}=\left(u_{0}, y_{0}\right)^{T} \in \mathcal{W}:=\mathcal{U} \times \mathcal{Y}$ a pair $\left(w_{1}, w_{2}\right)=$ $\left(\left(u_{1}, y_{1}\right)^{T},\left(u_{2}, y_{2}\right)^{T}\right) \in \mathcal{W}_{a} \times \mathcal{W}_{a}, \mathcal{W}_{a}:=\mathcal{U}_{a} \times \mathcal{Y}_{a}$, is a solution if, and only if, (1.1) holds on $\operatorname{dom}\left(w_{1}, w_{2}\right)$.

Let $\mathcal{X}_{w_{0}}:=\left\{\left(w_{1}, w_{2}\right) \in \mathcal{W}_{a}^{2} \mid\left(w_{1}, w_{2}\right)\right.$ solves $\left.(1.1)\right\}$ be the set of all solutions, which may be empty. The closed loop system $[P, C]$ is said to have the existence property, if $\mathcal{X}_{w_{0}} \neq \emptyset$ for all $w_{0} \in \mathcal{W}$, and the uniqueness property, if for all $w_{0} \in \mathcal{W}$ and $\left(\hat{w}_{1}, \hat{w}_{2}\right),\left(\tilde{w}_{1}, \tilde{w}_{2}\right) \in \mathcal{X}_{w_{0}}$, we have $\left(\hat{w}_{1}, \hat{w}_{2}\right)=\left(\tilde{w}_{1}, \tilde{w}_{2}\right) \quad$ on $\operatorname{dom}\left(\hat{w}_{1}, \hat{w}_{2}\right) \cap \operatorname{dom}\left(\tilde{w}_{1}, \tilde{w}_{2}\right)$.

Throughout the paper we assume that all closed loop systems under consideration satisfy the uniqueness property (but not necessarily the existence property).

For each $w_{0} \in \mathcal{W}$, define $\omega_{w_{0}} \in \mathcal{T} \cup\{\infty\}$, by the property $\left[0, \omega_{w_{0}}\right):=\bigcup_{\left(\hat{w}_{1}, \hat{w}_{2}\right) \in \mathcal{X}_{w_{0}}} \operatorname{dom}\left(\hat{w}_{1}, \hat{w}_{2}\right)$ and define $\left(w_{1}, w_{2}\right) \in \mathcal{W}_{a} \times \mathcal{W}_{a}$, with $\operatorname{dom}\left(w_{1}, w_{2}\right)=\left[0, \omega_{w_{0}}\right)$, by the property $R_{t}\left(w_{1}, w_{2}\right) \in \mathcal{X}_{w_{0}}$ for all $t \in\left[0, \omega_{w_{0}}\right)$. This induces the operator

$$
H_{P, C}: \mathcal{W} \rightarrow \mathcal{W}_{a} \times \mathcal{W}_{a}, \quad w_{0} \mapsto\left(w_{1}, w_{2}\right) .
$$

For $\Omega \subset \mathcal{W}$ the closed loop system $[P, C]$ given by (1.1), is said to be:

- locally well posed on $\Omega$ if, and only if, it has the existence and uniqueness properties and the operator $\left.H_{P, C}\right|_{\Omega}: \Omega \rightarrow \mathcal{W}_{a} \times \mathcal{W}_{a}, w_{0} \mapsto\left(w_{1}, w_{2}\right)$, is causal;

- globally well posed on $\Omega$ if, and only if, it is locally well posed on $\Omega$ and $H_{P, C}(\Omega) \subset \mathcal{W}_{e} \times \mathcal{W}_{e}$.

For the plant operator $P$ and the controller operator $C$ define the graph $\mathcal{G}_{P}$ of the plant and the graph $\mathcal{G}_{C}$ of the controller, respectively, as follows:

$$
\begin{aligned}
& \mathcal{G}_{P}:=\left\{\left(\begin{array}{c}
u \\
P u
\end{array}\right) \mid u \in \mathcal{U}, P u \in \mathcal{Y}\right\} \subset \mathcal{W}, \\
& \mathcal{G}_{C}:=\left\{\left(\begin{array}{c}
C y \\
y
\end{array}\right) \mid C y \in \mathcal{U}, y \in \mathcal{Y}\right\} \subset \mathcal{W} .
\end{aligned}
$$

Next define the operators

$$
\Pi_{P / / C}: \mathcal{W} \rightarrow \mathcal{W}_{a}, \quad w_{0} \mapsto w_{1},
$$

and

$$
\Pi_{C / / P}: \mathcal{W} \rightarrow \mathcal{W}_{a}, \quad w_{0} \mapsto w_{2}
$$

Clearly, $H_{P, C}=\left(\Pi_{P / / C}, \Pi_{C / / P}\right)$ and $\Pi_{P / / C}+\Pi_{C / / P}=I$.

For normed signal spaces $\mathcal{X}, \mathcal{V}$ and $\Omega \subset \mathcal{X}$ define the following:

(i) A causal operator $Q: \mathcal{X} \rightarrow \mathcal{V}_{a}$ is called gain stable on $\Omega$ if, and only if, $Q(\Omega) \subset \mathcal{V}, Q(0)=0$ and

$$
\left\|\left.Q\right|_{\Omega}\right\|_{\mathcal{X}, \mathcal{V}}:=\sup _{\substack{\tau>0, x \in \Omega \\ R_{\tau} x \neq 0}} \frac{\left\|R_{\tau} Q x\right\|_{\tau}}{\left\|R_{\tau} x\right\|_{\tau}}<\infty .
$$

(ii) A causal operator $Q: \mathcal{X} \rightarrow \mathcal{V}_{a}$ is called $(\gamma, \beta)$ gain stable with bias on $\Omega$ if, and only if, $Q(\Omega) \subset \mathcal{V}$ and

$$
\left\|R_{\tau} Q x\right\|_{\tau} \leq \gamma\left\|R_{\tau} x\right\|_{\tau}+\beta, \quad \forall x \in \Omega, \tau>0 .
$$

(iii) A causal operator $Q: \mathcal{X} \rightarrow \mathcal{V}_{a}$ is called gain stable with uniform bias on $\Omega$ if, and only if, $Q(\Omega) \subset \mathcal{V}$ and there exists $\beta \geq 0$ such that for all $R>0$ there exists $\gamma(R)>0$ such that:

$$
\left\|R_{\tau} Q x\right\|_{\tau} \leq \gamma(R)\left\|R_{\tau} x\right\|_{\tau}+\beta
$$

for all $x \in \Omega \cap B_{R}, \tau>0$.

(iv) A causal operator $Q: \mathcal{X} \rightarrow \mathcal{V}_{a}$ is called gain-function stable on $\Omega$ (or gf-stable on $\Omega$ ) if, and only if, $Q(\Omega) \subset \mathcal{V}$ and the nonlinear gain-function

$$
\begin{aligned}
g\left[\left.Q\right|_{\Omega}\right] & :\left(r_{0}, \infty\right) \rightarrow[0, \infty), \\
r & \mapsto g\left[\left.Q\right|_{\Omega}\right](r):=\sup _{\substack{x \in \Omega, \tau>0 \\
\left\|R_{\tau} x\right\|_{\tau} \in\left(r_{0}, r\right]}}\left\|R_{\tau} Q x\right\|_{\tau},
\end{aligned}
$$

is defined, where $r_{0}:=\inf _{x \in \Omega}\|x\|_{\mathcal{X}}<\infty$.

It is important to observe that in contrast to some treatments of gain functions, we do not require $g[Q](0)=0$ (if $r_{0}=0$ ).

Definition 2.1: Let $R \geq 0$. A causal operator $F: \mathcal{X}_{1} \rightarrow \mathcal{X}_{2}$ between normed spaces $\mathcal{X}_{1}, \mathcal{X}_{2}$ which is gain stable with 
uniform bias is said to have gain $\gamma_{R}[F]$ and bias $\beta_{R}[F]$ defined as:

$$
\left.\begin{array}{l}
\beta_{R}[F]=\inf \left\{\beta \geq 0 \mid \begin{array}{l}
\exists \gamma \geq 0 \text { such that } \\
\left\|T_{\tau} F x\right\| \leq \gamma\left\|T_{\tau} x\right\|+\beta, \\
\forall x \in \mathcal{X}_{1} \cap B_{R}, \forall \tau \geq 0
\end{array}\right\}, \\
\gamma_{R}[F]=\inf _{\varepsilon>0} \inf \left\{\gamma \geq 0 \mid \begin{array}{c}
\left\|T_{\tau} F x\right\| \leq \gamma\left\|T_{\tau} x\right\| \\
+\beta_{R}[F]+\varepsilon, \\
\forall x \in \mathcal{X}_{1} \cap B_{R}, \tau \geq 0
\end{array}\right.
\end{array}\right\} .
$$

For normed signal spaces $\mathcal{U}, \mathcal{Y}$ and $\mathcal{W}:=\mathcal{U} \times \mathcal{Y}$ and the causal operator $P: \mathcal{U}_{a} \rightarrow \mathcal{Y}_{a}$ and $C: \mathcal{Y}_{a} \rightarrow \mathcal{U}_{a}$ define the following:

(i) The closed-loop system $[P, C]$ given by (1.1) with the associated operator $\Pi_{P / / C}: \mathcal{W} \rightarrow \mathcal{W}_{a}$ is said to be BIBO $\mathcal{W}$-stable if, and only if, it is globally well posed and $H_{P, C}(\mathcal{W}) \subset \mathcal{W} \times \mathcal{W}$.

(ii) Let $\Omega \subset \mathcal{W}$. The closed-loop system $[P, C]$ given by (1.1) with the associated operator $\Pi_{P / / C}: \mathcal{W} \rightarrow \mathcal{W}_{a}$ is said to be gain stable (with (uniform) bias) on $\Omega$ if, and only if, $\Pi_{P / / C}$ is gain stable (with (uniform) bias) on $\Omega$.

(iii) Let $\Omega \subset \mathcal{W}$. The closed-loop system $[P, C]$ given by (1.1) with the associated operator $\Pi_{P / / C}: \mathcal{W} \rightarrow \mathcal{W}_{a}$ is said to be gain-function stable on $\Omega$ if, and only if, $\Pi_{P / / C}$ is gain-function stable on $\Omega$.

For all the above stability definitions, if an object is (gain) stable (with bias) on $\Omega=\mathcal{W}$, then it is said to be globally (gain) stable (with bias).

The notion of gain function stability and gain stability with uniform bias as closely related as the following lemma shows:

Lemma 2.2: The following statements are equivalent:

1) $[P, C]$ is gain function stable.

2) $[P, C]$ is gain stable with uniform bias.

\section{GAP DistanCES AND ROBUST PERFORMANCE MARGINS}

Throughout this paper, our principal measure of performance of a closed loop system $[P, C]$ is given by:

$$
A_{P, C}(r):=\sup _{\left\|w_{0}\right\| \leq r}\left\|\Pi_{P / / C} w_{0}\right\| .
$$

Let $\Gamma$ denote a set of causal operators $\mathcal{U}_{a} \rightarrow \mathcal{Y}_{a}$ corresponding to the admissible plants. Given $P, P_{1} \in \Gamma$ and a distance measure $\vec{\delta}: \Gamma \times \Gamma \rightarrow[0, \infty]$, the robust stability margin is defined as follows:

$$
B_{P, C}(r)=\sup \left\{\varepsilon>0 \mid \vec{\delta}\left(P, P_{1}\right)<\epsilon, A_{P_{1}, C}(r)<\infty\right\}
$$

and the robust performance margin is defined to be:

$$
\begin{aligned}
A B_{P, C}(r, \eta)=\sup \left\{\varepsilon>0 \mid \vec{\delta}\left(P, P_{1}\right)<\epsilon,\right. \\
\\
\left.A_{P_{1}, C}(r) \leq(1+\eta) A_{P, C}(r)\right\}
\end{aligned}
$$

\section{A. The Linear Gap}

We let $\mathcal{R}(\mathcal{U}, \mathcal{Y})$ denote the set of linear operators $P: \mathcal{U}_{e} \rightarrow$ $\mathcal{Y}_{e}$ specified by rational transfer functions. $\mathcal{H}^{\infty}$ denotes the Banach space of complex valued functions that are analytic and bounded on the open half plane $\mathbb{C}_{+}$with norm: $\|f\|_{\mathcal{H}^{\infty}}=$ $\operatorname{ess}_{\sup _{\omega \in \mathbb{R}}}|f(j \omega)| . \mathcal{R H}^{\infty}$ is the subset of $\mathcal{H}^{\infty}$ consisting of rational functions. The pair $N, D \in \mathcal{R H}^{\infty}$ are said to be normalized right co-prime factors over $\mathcal{R H}^{\infty}$ of a transfer function $P$, if $P=N D^{-1}$ and $N^{*} N+D^{*} D=I$. The set of all such $(N, D)$ is denoted by $\operatorname{NRCF}(P)$.

For the case of $\Gamma=\mathcal{R}\left(L^{2}\left(\mathbb{R}_{+}\right), L^{2}\left(\mathbb{R}_{+}\right)\right), C \in$ $\mathcal{R}\left(L^{2}\left(\mathbb{R}_{+}\right), L^{2}\left(\mathbb{R}_{+}\right)\right)$and we let $\vec{\delta}_{0}$ denote the directed $H_{2}$ gap metric:

$$
\vec{\delta}_{0}\left(P_{1}, P_{2}\right)=\underset{\left(\begin{array}{c}
\Delta_{N} \\
\Delta_{D}
\end{array}\right) \in \nabla}{ }\left\|\left(\begin{array}{c}
\Delta_{N} \\
\Delta_{D}
\end{array}\right)\right\|_{\mathcal{H}^{\infty}} .
$$

where $\nabla$ is the set of all $\left(\begin{array}{c}\Delta_{N} \\ \Delta_{D}\end{array}\right) \in \mathcal{R} \mathcal{H}^{\infty}$ such that $P_{2}=$ $\left(N_{1}+\Delta_{N}\right)\left(D_{1}+\Delta_{D}\right)^{-1}$ and $\left(N_{1}, D_{1}\right) \in \operatorname{NRCF}\left(P_{1}\right)$.

It is well known that $A_{P, C}(r)=b_{P, C}^{-1} r, B_{P, C}(r)=b_{P, C}$, where $b_{P, C}=\left\|\Pi_{P / / C}\right\|^{-1}$. If

$$
\vec{\delta}\left(P, P_{1}\right) \leq \varepsilon \quad \text { with } \varepsilon \leq \frac{\eta b_{P, C}}{1+b_{P, C}+\eta}, \eta \geq 0,
$$

then

$$
\varepsilon<b_{P, C} \text { and } \frac{1+\varepsilon}{1-\varepsilon\left\|\Pi_{P / / C}\right\|} \leq 1+\eta
$$

By Theorem 1 of [8], we have

$$
\left\|\Pi_{P_{1} / / C}\right\| \leq \frac{1+\varepsilon}{1-\varepsilon\left\|\Pi_{P / / C}\right\|}\left\|\Pi_{P / / C}\right\| \leq(1+\eta)\left\|\Pi_{P / / C}\right\| .
$$

This shows $A_{P_{1}, C}(r) \leq(1+\eta) A_{P, C}(r)$. Hence

$$
A B_{P, C}(r, \eta) \geq \frac{\eta b_{P, C}}{1+b_{P, C}+\eta} \text { for all } \eta, r \geq 0 \text {. }
$$

\section{B. The Un-Biased Regional Nonlinear Gap}

Let $\Gamma$ denote the set of all causal operators $\mathcal{U}_{a} \rightarrow \mathcal{Y}_{a}$. Given $P_{1}, P_{2} \in \Gamma$ and a subset $\Omega \subset \mathcal{V}$ of a complete normed signal space $\mathcal{V}$, define the (possibly empty) set

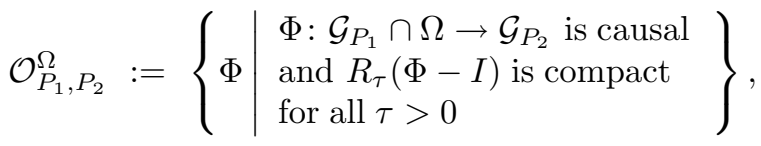

and the nonlinear directed gap [8]:

$$
\vec{\delta}_{\Omega}\left(P_{1}, P_{2}\right)= \begin{cases}\ell & \text { if } \mathcal{O}_{P_{1}, P_{2}}^{\Omega} \neq \emptyset \\ \infty & \text { if } \mathcal{O}_{P_{1}, P_{2}}^{\Omega}=\emptyset\end{cases}
$$

where

$$
\ell=\inf _{\Phi \in \mathcal{O}_{P_{1}, P_{2}}^{\Omega}} \sup _{\substack{x \in \mathcal{G}_{P_{1}} \cap \Omega, \tau>0 \\ R_{\tau} x \neq 0}} \frac{\left\|\left.R_{\tau}(\Phi-I)\right|_{\mathcal{G}_{P_{1}}} x\right\|_{\tau}}{\left\|R_{\tau} x\right\|_{\tau}} .
$$

Various equivalent expressions for the (global) nonlinear gap can be found in [1], [9], including formulae based on nonlinear coprime factorisations closely related in form to (3.1). The $L^{2}\left(\mathbb{R}_{+}\right)$nonlinear gap is a generalisation of the standard definition of the $H_{2}$ gap $\vec{\delta}_{0}(\cdot, \cdot)$ [8], [11], in the sense that if $P_{1}, P_{2} \in \mathcal{R}\left(L^{2}\left(\mathbb{R}_{+}\right), L^{2}\left(\mathbb{R}_{+}\right)\right)$, and either $\vec{\delta}_{0}\left(P_{1}, P_{2}\right)<1$ or $\vec{\delta}_{0}\left(P_{2}, P_{1}\right)<1$, then it is shown in [7, Proposition 5] that if $\Omega=B_{R}:=\left\{w \in L^{2}\left(\mathbb{R}_{+}\right) \mid\|w\|_{L^{2}\left(\mathbb{R}_{+}\right)} \leq R\right\}, R>0$, then $\vec{\delta}_{0}\left(P_{1}, P_{2}\right)=\vec{\delta}_{\Omega}\left(P_{1}, P_{2}\right)$.

For nonlinear systems, we have $A_{P, C}(r) \leq\left\|\left.\Pi_{P / / C}\right|_{B_{r}}\right\| r$. However, in contrast to the linear setting, the robust stability margin is also in general dependent on the disturbance level 
$r>0$, and the parallel projection gain only provides a lower bound:

$$
\begin{aligned}
B_{P, C}(r) & \geq\left(\sup _{0<\left\|\left(u_{0}, y_{0}\right)\right\| \leq R} \frac{\left\|\Pi_{P / / C}\left(u_{0}, y_{0}\right)\right\|}{\left\|\left(u_{0}, y_{0}\right)\right\|}\right)^{-1} \\
& =:\left\|\left.\Pi_{P / / C}\right|_{B_{R}}\right\|^{-1}
\end{aligned}
$$

for some appropriate choice of $R>r$ see [8]. Furthermore, neither reverse inequality necessarily hold. So the robust performance margin $A B_{P, C}(r, \eta)$ for general nonlinear systems cannot be estimated as simply as in the linear system case. However, if $A_{P, C}(r)$ satisfies certain growth assumptions, then we are still able to estimate $A B_{P, C}(r, \eta)$ as shown in the following lemma.

Lemma 3.1: Suppose that 1) for $r>0$ and $\varepsilon \in$ $\left(0,\left\|\Pi_{P / / C} \mid \Omega\right\|^{-1}\right)$, there exists $R \geq r$ such that

$$
B_{R} \subset \Omega \text { and }\left\|\left.\Pi_{P / / C}\right|_{B}\right\| \leq \frac{R-r}{R \varepsilon} ;
$$

2) there exists a function $f:[0, \infty) \times(1, \infty) \rightarrow[0, \infty)$ such that

$$
A_{P, C}(k r) \leq f(r, k) A_{P, C}(r) \text { for } k, r \geq 0 .
$$

Then

$$
\begin{aligned}
& A B_{P, C}(r, \eta) \geq \\
& \sup _{\varepsilon>0}\left\{(1+\varepsilon) f\left(r, \frac{1}{1-\varepsilon\left\|\left.\Pi_{P / / C}\right|_{B_{R}}\right\|}\right) \leq 1+\eta\right\} .
\end{aligned}
$$

Note that for linear systems, assumptions 1) and 2) are satisfied with $R=1 /\left(1-\varepsilon\left\|\Pi_{P / / C}\right\|\right)$ and $f(r, k)=k$, and inequality (3.7) reduce to inequality (3.2).

It follows that the minimization of $\left\|\left.\Pi_{P / / C}\right|_{B_{r}}\right\|$ remains, as in the LTI case, a sensible design objective, since a small $\left\|\left.\Pi_{P / / C}\right|_{B_{r}}\right\|$ ensures both a good robust stability margin and good nominal performance. However, as we will see next, it is not always possible to achieve $\left\|\left.\Pi_{P / / C}\right|_{B_{R}}\right\|<\infty$, even when a sensible robust stability margin should exist.

\section{A MOTIVATING EXAMPLE}

The potential lack of tightness of the lower bound (3.5) is not pathological [3], [4]; many adaptive controllers have the property

$$
B_{P, C}(r)>0 \text { for all } r \geq 0
$$

whilst

$$
\sup _{\left\|u_{0}, y_{0}\right\| \leq r}\left(\frac{\left\|\Pi_{P / / C}\left(u_{0}, y_{0}\right)\right\|}{\left\|\left(u_{0}, y_{0}\right)\right\|}\right)=\infty \quad \text { for all } r>0 .
$$

This arises due to a problem with small signal behaviour, where whilst $\Pi_{P / / C}(0)=0$, the operator $\Pi_{P / / C}$ is not continuous at $0-$ which precludes the existence of a 'local finite gain'.

An explicit example of this (in an $L^{2}$ setting) is given by the plant

$$
P(\theta)\left(u_{1}\right)=y_{1} \text { where } \dot{y}_{1}=\theta y_{1}+u_{1} y_{1}(0)=0,
$$

with $\theta>0$ and the controller:

$$
\begin{aligned}
C\left(y_{2}\right)(t) & =u_{2}(t) \\
u_{2}(t) & =-k(t)^{\frac{1}{4}} y_{2}(t) \\
\dot{k}(t) & =y_{2}^{2} .
\end{aligned}
$$

It has been shown that this closed loop is BIBO stable in an $L^{2}\left(\mathbb{R}_{+}\right)$setting, see [4]. Clearly $u_{0}=y_{0}=0$ implies $u_{1}, y_{1}=0$, ie. $\Pi_{P / / C}(0)=0$, but for any disturbance (arbitrarily small) which moves $y_{1} \neq 0$, the system is unstable unless there exists a time at which $k(t) \geq \theta^{4}$, ie. $\left\|y_{2}\right\|_{L^{2}[0, t]} \geq$ $\theta^{2}$. Hence for all $\epsilon>0, \exists u_{0}, y_{0},\left\|\left(u_{0}, y_{0}\right)\right\| \leq \epsilon$

$$
\left\|\Pi_{C / / P}\right\| \geq \frac{\left\|\left(u_{2}, y_{2}\right)^{T}\right\|}{\left\|\left(u_{0}, y_{0}\right)^{T}\right\|} \geq \frac{\theta^{2}}{\epsilon} \rightarrow \infty \quad \text { as } \epsilon \rightarrow 0 .
$$

Hence $\left\|\Pi_{P / / C}\right\|=\infty$, and this is caused by a lack of continuity at 0 .

However, let us first note that this discontinuity is addressed in [4] by appending $\theta$ onto the input space, for then an inequality of the form:

$$
\left\|\left(u_{1}, y_{1}, \theta\right)\right\|_{\mathcal{U} \times \mathcal{Y} \times \mathbb{R}} \leq g\left(\left\|\left(u_{0}, y_{0}\right)\right\|_{\mathcal{U} \times \mathcal{Y}},|\theta|\right),
$$

was constructed, and from this it was shown in [4] that $B_{P(\theta), C}(r)>0$, ie. we have a non zero but disturbance dependent robustness margin. Hence both (4.1) and (4.2) hold.

Similar conclusions apply if we take the more standard adaptive controller with $u_{2}(t)=-k(t) y_{2}(t)$ in (4.4), since an inequality of the form (4.5) was established in [6], from which the construction of [4] similarly yields $B_{P(\theta), C}(r)>0$. Similar conclusions also hold in a MIMO setting [6].

\section{REGIONAL ROBUST STABILITY}

In the context of a notion of stability with bias, it is natural to adopt this notion of stability to assess the 'gap' between the graphs of $P$ and $P_{1}$. Hence for $\Omega \subset \mathcal{W}$ define the set:

$$
\begin{aligned}
& \vec{\Delta}\left(P_{1}, P_{2} ; \Omega\right)=\left\{(\vec{\delta}, \vec{\sigma}) \in \mathbb{R}_{+}^{2}: \exists \Phi \in \mathcal{O}_{P_{1}, P_{2}}^{\Omega}\right. \text { s.t. } \\
& \left.\left\|R_{\tau}(I-\Phi) x\right\| \leq \vec{\delta}\|x\|_{\tau}+\vec{\sigma}, \forall x \in \mathcal{G}_{P_{1}} \cap \Omega\right\}
\end{aligned}
$$

where $\mathcal{O}_{P_{1}, P_{2}}^{\Omega}$ is given by (3.3). Thus the set $\vec{\Delta}\left(P_{1}, P_{2} ; \Omega\right)$ consists of all possible gains and biases which describe the deviation from the identity of maps $\Phi$ between the graphs of $P$ and $P_{1}$ restricted to $\Omega$. In contrast to the nonlinear gap (3.4), there are now two parameters $\vec{\delta}, \vec{\sigma}$ describing this 'gap', and there is no natural way to reduce this to a single distance measure. Since there are different ways of defining a distance measure, see Section 6 below, we elect to present the main regional robust stability result without making such choices, i.e. the description of the 'gap' between $P_{1}$ and $P_{2}$ remains described by the two dimensional set $\Delta\left(P_{1}, P_{2} ; \Omega\right)$ of all possible gains and biases.

In the rest of this paper, unless specified otherwise, we always let $\mathcal{U}, \mathcal{Y}$ be truncation complete normed signal spaces, let $\mathcal{W}=\mathcal{U} \times \mathcal{Y}$, and suppose that for all $\tau>0$, there exists a continuous mapping $E_{\tau}: W[0, \tau] \rightarrow W$ such that

$$
R_{\tau} x=R_{\tau}\left(E_{\tau} x\right), \text { for all } x \in W[0, \tau) .
$$

An operator $Q: \mathcal{W} \rightarrow \mathcal{W}$ is said to be relatively continuous if for all $\tau>0$ and for all operators $\Psi: \mathcal{W} \rightarrow \mathcal{W}$ with $R_{\tau} \Psi$ compact, the operator $R_{\tau} \Psi Q: \mathcal{W} \rightarrow \mathcal{W}[0, \tau)$ is continuous.

Theorem 5.1: Consider $P: \mathcal{U}_{a} \rightarrow \mathcal{Y}_{a}, P_{1}: \mathcal{U}_{a} \rightarrow \mathcal{Y}_{a}$ and $C: \mathcal{Y}_{a} \rightarrow \mathcal{U}_{a}$. Let $R>0,0<\epsilon<1$. Suppose $(\vec{\delta}, \vec{\sigma}),(\gamma, \beta) \epsilon$ $\mathbb{R}_{+}^{2}$ are such that:

$$
(\vec{\delta}, \vec{\sigma}) \in \Delta\left(P, P_{1} ; \Omega\right)
$$


where $\Omega=B\left(\gamma \frac{\rho(\epsilon R)}{\epsilon}+\beta\right) \subset \mathcal{W}, \rho(R)=R+\vec{\sigma}+\vec{\delta} \beta$, $[P, C]$ is $(\gamma, \beta)$ gain stable with bias on $B\left(\frac{\rho(\epsilon R)}{\epsilon}\right) \subset \mathcal{W}$ and $\Pi_{P, C}$ is relatively continuous. If $\left[P_{1}, C\right]$ has the uniqueness property and

$$
\vec{\delta} \gamma<1-\epsilon
$$

then the closed-loop system $\left[P_{1}, C\right]$ is $\left(\gamma_{1}, \beta_{1}\right)$ gain stable with bias on $B(\epsilon R)$ and

$$
\gamma_{1}=\gamma \frac{1+\vec{\delta}}{\epsilon}, \quad \beta_{1}=\beta+(\vec{\sigma}+\vec{\delta} \beta)\left(1+\gamma \frac{1+\vec{\delta}}{\epsilon}\right) \text {. }
$$

We remark that if the operator $R_{\tau} \Phi$ used to define $Q_{w}$ is locally incrementally stable, i.e.

$$
\sup _{\substack{R_{\tau} w_{1} \neq R_{\tau} w_{2} \\\left\|R_{\tau} w_{1}\right\|,\left\|R_{\tau} w_{2}\right\| \leq r}} \frac{\left\|R_{\tau} \Phi w_{1}-R_{\tau} \Phi w_{1}\right\|_{\tau}}{\left\|R_{\tau} w_{1}-R_{\tau} w_{2}\right\|_{\tau}}<\infty
$$

then the relative continuity requirement for $\Pi_{P, C}$ can be replaced by that $R_{\tau} \Pi_{P, C}$ is continuous.

\section{ROBUST STABILITY AND PERFORMANCE}

We have already observed that in Theorem 5.1 the set $\Delta\left(P, P_{1} ; \Omega\right) \subset \mathbb{R}_{+}^{2}$ plays the role of the gap distance in the unbiased robust stability theorem, and that as a 2-dimensional description of the 'gap' between $P$ and $P_{1}$, it does not define a distance between the two plants. Some possible scalar measures are:

1) Gap defined with respect to the smallest gain:

$$
\begin{aligned}
& \vec{\delta}_{R}\left(P_{1}, P_{2}\right) \\
& =\inf \left\{\vec{\delta} \geq 0 \mid \exists \vec{\beta} \text { s.t. }(\vec{\delta}, \vec{\beta}) \in \vec{\Delta}\left(P_{1}, P_{2} ; B_{R}\right)\right\} .
\end{aligned}
$$

Correspondingly we define the bias:

$$
\begin{aligned}
\vec{\beta}_{R}\left(P_{1}, P_{2}\right) & =\inf \{\vec{\beta} \geq 0 \mid \exists \vec{\beta} \text { s.t. } \\
& \left.\left(\vec{\delta}_{R}\left(P_{1}, P_{2}\right), \vec{\beta}\right) \in \overrightarrow{\vec{\Delta}\left(P_{1}, P_{2} ; B_{R}\right)}\right\} .
\end{aligned}
$$

2) Gap defined with respect to the smallest bias:

$$
\vec{\delta}_{R}\left(P_{1}, P_{2}\right)=\inf \left\{\vec{\delta} \geq 0 \mid\left(\vec{\delta}, \vec{\beta}_{R}\right) \in \overline{\vec{\Delta}\left(P_{1}, P_{2} ; B_{R}\right)}\right\}
$$

where

$$
\vec{\beta}_{R}=\inf \left\{\vec{\beta} \geq 0 \mid \exists \vec{\delta} \text { s.t. }(\vec{\delta}, \vec{\beta}) \in \vec{\Delta}\left(P_{1}, P_{2} ; B_{R}\right)\right\} .
$$

3) Gap defined at a given bias level $\vec{\beta}>0$ :

$$
\vec{\delta}_{R}\left(P_{1}, P_{2}\right)=\inf \left\{\vec{\delta} \geq 0 \mid(\vec{\delta}, \vec{\beta}) \in \vec{\Delta}\left(P_{1}, P_{2} ; B_{R}\right)\right\} .
$$

Note that $\vec{\delta}_{R}\left(P_{1}, P_{2}\right)$ at bias level $\beta=0$ recovers the nonlinear gap (3.4).

We now specialize to the case where the gap between the plant can be described without biases as in (3.4), which incorporates the case of linear plants in $\mathcal{R}\left(L^{2}\left(\mathbb{R}_{+}\right), L^{2}\left(\mathbb{R}_{+}\right)\right)$. That is, in the following, $\vec{\delta}$ is given by (3.4) with $\Omega=\mathcal{W}$. This is a special case of both 2 . and 3. above. This special case is important for the adaptive control setting considered in sections 4 and 7, where the plant is linear, hence the gap can be measured by $\vec{\delta}_{0}$, and the bias arises from the nonlinear controller. As the $P$ and $P_{1}$ do have a finite nonlinear gap then this coincides with the distance measure in 2. above, whilst in relation to case 3 . we are just considering the gap at bias level 0 .

Corollary 6.1: Suppose $[P, C]$ is $(\gamma(\cdot), \beta)$ gain stable with uniform bias and $\Pi_{P / / C}$ is relatively continuous. Let $0<\epsilon<$ 1. If $\left[P_{1}, C\right]$ has the uniqueness property and

$$
\vec{\delta}\left(P, P_{1}\right) \gamma\left(\frac{R+\vec{\delta}\left(P, P_{1}\right) \beta}{\epsilon}\right)<1-\epsilon
$$

then the closed-loop system $\left[P_{1}, C\right]$ is $\left(\gamma_{1}(R), \beta_{1}\right)$ gain stable with bias on $B(R)$ where:

$$
\begin{aligned}
& \gamma_{1}(R)=\gamma\left(\frac{R+\vec{\delta}\left(P, P_{1}\right) \beta}{\epsilon}\right)\left(\frac{1+\vec{\delta}\left(P, P_{1}\right)}{\epsilon}\right), \\
& \beta_{1}=\beta\left(1+\vec{\delta}\left(P, P_{1}\right) \beta \mu\right) \text { with } \\
& \mu=1+\gamma\left(\frac{R+\vec{\delta}\left(P, P_{1}\right) \beta}{\epsilon}\right) \frac{1+\vec{\delta}\left(P, P_{1}\right)}{\epsilon} .
\end{aligned}
$$

Corollary 6.2: Consider $P, P_{1} \in \mathcal{R}(\mathcal{U}, \mathcal{Y})$. Suppose $[P, C]$ is $(\gamma(\cdot), \beta)$ gain stable with uniform bias. Then:

$$
B_{P, C}(R) \geq \sup _{\epsilon \in(0,1)}\left\{r>0 \mid r \gamma\left(\frac{R+r \beta}{\epsilon}\right)<1-\epsilon\right\} .
$$

Corollary 6.3: Suppose $[P, C]$ is $(\gamma(\cdot), \beta)$ gain stable with uniform bias and $\Pi_{P / / C}$ is relatively continuous. Suppose that

1) for $r>0$ and $\varepsilon \in\left(0, B_{P, C}(r)\right)$, there exists $R$ such that

$$
r+\varepsilon(\gamma(R) R+\beta) \leq R
$$

2) there exists a function $f:[0, \infty) \times(1, \infty) \times(1, \infty) \rightarrow$ $[0, \infty)$ such that for all $r \geq 0, k_{1}, k_{2} \geq 1$

$$
A_{P, C}\left(k_{1} r+k_{2}\right) \leq f\left(r, k_{1}, k_{2}\right) A_{P, C}(r)
$$

Then

$$
A B_{P, C}(r, \eta) \geq \sup \{\varepsilon \geq 0: \varepsilon \text { satisfies (6.2) below }\},
$$

$$
(1+\varepsilon) f\left(r, \frac{1}{1-\varepsilon \gamma(R)}, \frac{\varepsilon \beta}{1-\varepsilon \gamma(R)}\right) \leq 1+\eta .
$$

If 2) is replaced by

2') $r \mapsto r-\varepsilon(\gamma(r) r+\beta)$ is invertible with a non-decreasing inverse $h_{\varepsilon, \beta}(r)$ and there exists a function $g: \mathbb{R}_{+}^{3} \rightarrow[0, \infty)$ such that for all $r \geq 0$

$$
A_{P, C}\left(h_{\varepsilon, \beta}(r)\right) \leq g(r, \varepsilon, \beta) A_{P, C}(r)
$$

Then

$$
A B_{P, C}(r, \eta) \geq \sup \{\varepsilon \geq 0:(1+\varepsilon) g(r, \varepsilon, \beta) \leq 1+\eta\} .
$$

\section{ROBUST STABILITY FOR ADAPTIVE CONTROL}

Let us consider the adaptive controller (4.4) from Section 4.

From [4] it is known that this controller has closed loop stability properties for minimum phase, relative degree one linear plants with possible high frequency, in particular any plant of the form (4.3) for $\theta \in \mathbb{R}$.

In particular, it was shown in [4] that there exists a continuous function $g: \mathbb{R}_{+}^{2} \rightarrow \mathbb{R}_{+}$such that (4.5) holds, and it was further shown that BIBO stability was preserved for sufficiently small gap perturbations of $P(\theta)$. 
We now show that in fact gain stability with uniform bias is preserved for sufficiently small gap perturbations of $P(\theta)$. The explicit inequality (4.5) in [4] can be rearranged to see that for fixed $\theta, \Pi_{P(\theta) / / C}$ is gain stable with uniform bias:

$$
\left\|\Pi_{P(\theta) / / C} w_{0}\right\| \leq \gamma\left(\left\|w_{0}\right\|\right)\left\|w_{0}\right\|+\beta
$$

It now follows from Corollary 6.1 that gain stability with uniform bias is preserved for sufficiently small gap perturbations of $P(\theta)$ given by inequality (6.1). $B_{P, C}(r), A B_{P, C}(r)$ can be obtained from Corollaries 6.2 and 6.3. As in Section 4, similar conclusions apply (also in the MIMO setting) if we take the more standard adaptive controller with $u_{2}(t)=-k(t) y_{2}(t)$ in (4.4), since an inequality of the form (4.5) was also established in [6].

\section{Global Robust Stability}

By applying Theorem 5.1 in a global setting we obtain the following global result:

Theorem 8.1: Consider $P: \mathcal{U}_{a} \rightarrow \mathcal{Y}_{a}, P_{1}: \mathcal{U}_{a} \rightarrow \mathcal{Y}_{a}$ and $C: \mathcal{Y}_{a} \rightarrow \mathcal{U}_{a}$. Suppose $(\vec{\delta}, \vec{\sigma}) \in \Delta\left(P, P_{1} ; \mathcal{W}\right),[P, C]$ is $(\gamma, \beta)$ gain stable with bias on $\mathcal{W}$ and $\Pi_{P / / C}$ is relatively continuous. If $\left[P_{1}, C\right]$ has the uniqueness property and $\vec{\delta} \gamma<$ $1-\epsilon$ for some $\varepsilon \in(0,1)$, then the closed-loop system $\left[P_{1}, C\right]$ is $\left(\gamma_{1}, \beta\right)$ gain stable with bias on $\mathcal{W}$ and

$$
\gamma_{1}=\gamma \frac{1+\vec{\delta}}{\epsilon}, \beta_{1}=\beta+(\vec{\sigma}+\vec{\delta} \beta)\left(1+\gamma \frac{1+\vec{\delta}}{\epsilon}\right) \text {. }
$$

We remark that in contrast to the approach to derive the (unbiased) global results in [8], here we impose a compactness requirement in the definition of $\mathcal{O}_{P_{1}, P_{2}}^{\mathcal{W}}$. In turn this stronger requirement on the maps $\Phi$ results in substantially weaker assumptions on $\left[P_{1}, C\right]$. In [8] it was required that $\left[P_{1}, C\right]$ was globally well posed, and e.g. in [5] the alternative requirement of regularly well posed was used. Here, the only requirement is that $\left[P_{1}, C\right]$ satisfies the uniqueness property. This is weaker than either the assumption of global or regular well posedness, and is often straightforward to verify (in contrast to the existence property of well posedness assumptions which is often hard to verify a-priori).

\section{CONCLUDING REMARKS}

We have presented a generalisation to the nonlinear robust stability theory of Georgiou and Smith [8] which allows a notion of stability with bias terms. This approach contrasts to the alternative biased approach of [7] as it does not measure the gain w.r.t. a single offset 'bias' trajectory, and is applicable in situations where the need for a bias arises for other reasons, e.g. from a lack of continuity of $\Pi_{P / / C}$ at 0 as in the adaptive control example. This extension is significant for the provision of robust stability certificates for the many nonlinear systems for which no induced gain exists, and yet robust stability guarantees can still be given. We have illustrated this by an example from adaptive control where an induced gain does not exist.

We now draw attention to an significant difference between the nonlinear biased setting and the linear unbiased case. An important feature of the standard robust stability theory for LTI systems, is that the problem of maximizing the robust stability margin $B_{P, C}(r)$ is equivalent to minimizing the nominal performance $A_{P, C}(r)$, since $B_{P, C}(r)=\left\|\Pi_{P / / C}\right\|^{-1}=$ $r A_{P, C}^{-1}(r)$. Not only is this optimization tractable (in the $L^{2}$ setting minimizing $\left\|\Pi_{P / / C}\right\|^{-1}$ forms the standard $H_{\infty}$ problem), but it shows that optimizing nominal performance and the robust stability margin is equivalent.

This equivalence breaks down in the global nonlinear biased setting, as one can see that the robust stability margin $B_{P, C}(r)$ is maximised by minimising the nominal closed loop gain $\gamma\left[\Pi_{P / / C}\right]$, whereas the nominal performance, $A_{P, C}(r)$, is a function of both the gain $\gamma\left[\Pi_{P / / C}\right]$ and the bias $\beta\left[\Pi_{P / / C}\right]$. Thus optimizing the robust stability margin, i.e. by minimizing the gain term, will produce sub-optimal nominal performance. We can therefore conclude that robust stability and (nominal) performance constraints must both be specified in any sensible optimization.

Similar reasoning does not directly apply in the regional setting, since the situation is more complex: the bias determines the region over which the gain in computed, and hence the bias does affect the robust stability margin. However it is clear that the resulting optimizations are different, and hence the conclusion that nominal performance and robust stability margin optimization differ still holds. Thus any sensible formulation of an optimal robust design in this setting must independently specify requirements for both nominal performance and robust stability margins.

\section{REFERENCES}

[1] W. Bian and M. French. Graph topologies, gap metrics and robust stability for nonlinear systems. SIAM J. Control Optim., 44(2):418-443, 2005.

[2] C. Desoer and M. Vidyasagar.Feedback Systems Input Output Properties. Academic Press, 1st edition, 1975.

[3] M. French. Smooth adaptive controllers have discontinuous closed loop operators. In 16th Int. Symp. on Mathematical Theory of Networks and Systems, 2004. CD-Rom proceedings.

[4] M. French. Adaptive control and robustness in the gap metric. IEEE Trans. Autom. Control, 53(2):461-478, 2008.

[5] M. French, A. Ilchmann, and M. Müller. Robust stabilization by linear output delay feedback. SIAM J. Control Optim., 48(4): 2533-2561, 2009

[6] M. French, A. Ilchmann, and E.P. Ryan. Robustness in the graph topology of a common adaptive controller. SIAM J. Control Optim., 45(5):1736-1757, 2006.

[7] T.T. Georgiou and M.C. Smith. Biased norms and robustness analysis for nonlinear feedback systems. In Proc. of the 36th IEEE Conf. on Decision and Control, pages 642-643, 1997.

[8] T.T. Georgiou and M.C. Smith. Robustness analysis of nonlinear feedback systems. IEEE Trans. Autom. Control, 42(9):1200-1229, 1997

[9] M.R. James, M.C. Smith, and G. Vinnicombe. Gap metrics, representations and nonlinear robust stability. SIAM J. Control Optim., 43(5):1535-1582, 2005.

[10] M. Vidyasagar. Nonlinear Systems Analysis. Prentice Hall, 1993.

[11] G. Zames and A. K. El-Sakkary. Unstable systems and feedback: The gap metric. In Proc. of the Allerton Conf., pages 380-385, 1980. 